\title{
A Comparative Analysis of Impulse Noise Removal Techniques on Gray Scale Images
}

\author{
V. Murugan ${ }^{1}$, T. Avudaiappan ${ }^{2}$ and Dr. R. Balasubramanian ${ }^{3}$ \\ Research Scholar ${ }^{1}$, Department of Computer Science \& Engg, \\ Manonmaniam Sundaranar University, Tirunelveli - 627012, TamilNadu, India. \\ Research Scholar ${ }^{2}$, Professor ${ }^{3}$, Department of Computer Science \& Engg, \\ Manonmaniam Sundaranar University, Tirunelveli - 627012, TamilNadu, India. \\ Email:vmurugan@msuniv.ac.in, ${ }^{2}$ Email: avudaiappanmecse@gmail.com \\ ${ }^{3}$ rbalus662002@yahoo.com
}

\begin{abstract}
Various kinds of images and pictures are required as sources of information for analysis and interpretation. When an image is converted from one form to another such as scanning, transmitting, digitizing, storing etc., degradation occurs to the output image. Hence, the output image needs to be enhanced in order to be better analyzed. Denoising is the one of the pre processing technique in digital image processing. This paper investigates the performance of four denoising methods for removing the High Density Impulse Noise. They are Adaptive Bilateral Filter (ABF), Fuzzy Peer Group Filter (FPGF), Switching Bilateral Filter (SBF), and Boundary Discriminative Noise Detection Filter (BDND).The performance of the above four filters is compared by using five performance metrics. They are PeakSignal-to-Noise-Ratio, Mean Square Error and Root Mean Square Error. The Experimental results show that the BDND filter based denoising method performs well than the other three methods.
\end{abstract}

Keywords: Impulse Noise, Enhancement, filters, PSNR, MSE, SSIM, MAE and MD

\section{Introduction}

Image Enhancement is a low-level process, which involves reducing the Noise from images. Digital images are often corrupted by Noise during their acquisition and transmission [1]. In particular, most commonly known of Noise are called additive Gaussian Noise and Impulse Noise. The need for efficient image denoising methods has been grown with the massive production of digital images and movies of all kinds, often taken in poor conditions. In recent years, a new concept in edge-preserving de-noising was proposed by Tomasi and Manduchi [2]. Most filters for Gaussian Noise suppression are designed to take advantage of the zero-mean property of the Noise and try to suppress it by locally averaging pixel channel values. To approach this problem, many nonlinear methods has been recently proposed, for instance: the bilateral filter [2, 3], the anisotropic diffusion [4], the chromatic filter [5], or the soft switching methods in [6], which motivate other fuzzy methods as the fuzzy directional derivative filter [7], the fuzzy bilateral filter [8], the fuzzy Noise reduction method [9], or the fuzzy-switching filter [10].

Impulse Noise (Salt \& Pepper Noise):- 
Salt and pepper noise is a form of noise usually seen on images. It uniquely represents itself as randomly occurring white and black pixels. An effective noise reduction approach for this type of noise involves the usage of a median filter or a contrast harmonic mean filter. Salt and pepper noise affects into images in situations where the image is transferred quickly. The aim of these methods is to detect edges and details by means of local statistics and smooth them less than the rest of the image to better preserve their sharpness. However, these methods commonly identify Impulses as details or edges to be preserved, and, therefore, they are not able to reduce that this present system evaluate the performance of the image denoising techniques namely Adaptive Bilateral Filter (ABF) [11]. Fuzzy Peer Group Filter (FPGF) [12] Switching Bilateral Filter (SBF) [13] and BDND filter [14].

This paper is organized as follows: Section II presents an Adaptive bilateral filter, Fuzzy peer group filter, switching bilateral filter, BDND filter of image denoising methods. The results of application of the schemes are presented in section III Section IV presents conclusion of this paper.

\section{Algorithm}

\subsection{Adaptive Bilateral Filter [11]}

The response at $[\mathrm{m} 0, \mathrm{n} 0]$ of the proposed shift-variant $\mathrm{ABF}$ to an impulse at $[\mathrm{m}, \mathrm{n}]$ is given shown at the bottom of the page, where $[\mathrm{m} 0, \mathrm{n} 0]$ and $\Omega \mathrm{m} 0, \mathrm{n} 0$ are defined as before, and the normalization factor is given by

$$
\begin{gathered}
\gamma_{m 0, n 0}= \\
\sum_{m=m 0-N}^{m 0+N} \sum_{n=n 0-N}^{n 0+N} \exp \left(\frac{(m-m 0)^{2}+(n-n 0)^{2}}{2 \sigma^{2}}\right) \times \\
\exp \left(\frac{\left((g[m, n]-g[m 0, n 0]-\zeta[m 0, n 0])^{2}\right)}{2 \sigma^{2}[m 0, n 0]}\right)
\end{gathered}
$$

The ABF [11] retains the general form of a bilateral filter, but contains two important modifications. First, an offset $\zeta$ is introduced to the range filter in the ABF. Second, both $\zeta$ and the width of the range filter $\sigma_{r}$ in the ABF are locally adaptive. If $\zeta=0$ and $\sigma_{r}$ is fixed, the ABF will degenerate into a conventional bilateral filter. For the domain filter, a fixed lowpass Gaussian filter $\sigma_{r}=1.0$ with is adopted in the ABF. The combination of a locally adaptive $\zeta$ and $\sigma_{r}$ transforms the bilateral filter into a much more powerful filter that is capable of both smoothing and sharpening. Moreover, it sharpens an image by increasing the slope of the edges. To understand how the ABF works, we need to understand the role of $\zeta$ and $\sigma_{r}$ in the $\mathrm{ABF}$.

\subsection{Fuzzy Peer Group Filter [12]}

This paper [12] uses a fuzzy similarity function, as the function above which, following the above terminology, is given by

$$
\rho\left(F_{i} F_{j}\right)=e^{-\frac{\left\|F_{i-} F_{j}\right\|}{F_{(i)}}}, \mathrm{i}, \mathrm{j}=0 \ldots \ldots \ldots \ldots \mathrm{n}^{2}-1
$$

Where $\|$.$\| denotes the Euclidean norm and F_{\sigma}>0$

is a parameter. 


\subsection{Switching Bilateral Filter [13]}

A noise detector is used in the SBF [13] filter to determine whether or not the current pixel is corrupted. This decision is made using the features of sorted quadrant median vector (SQMV), which can show the property of the background and is more reliable than only one median value. We obtain the reference median for noise identification from SQMV. If the reference median is improper, it can lead to lost detection or over detection. The lost noisy pixels have a great negative effect on the results and the undesired filtering removes the details. When a current pixel is very different from the reference median, it is identified as an impulse noise. When the difference between the current pixel and reference median is not too much, it may be a Gaussian noise or a noise-free pixel. Because the image background can give four different SQM values, the reference median can be selected from SQMV. The decision making mechanism is realized by employing a reference median and two thresholds The switching bilateral filter is defined by using the below formula

$$
u_{i, j}=\frac{\sum_{S=-N}^{N} \sum_{t=-N}^{N} W_{G}(s, t) W_{S R}(s, t) x_{i+s j+t}}{\sum_{S=-N}^{N} \sum_{t=-N}^{N} W_{G}(s, t) W_{S R}(s, t)}
$$

$$
\begin{array}{r}
\text { Where } \\
W_{G}(s, t)=\exp -\frac{(i-s)^{2}+(j-t)^{2}}{2 \sigma^{2} S} \\
W_{S R}(s, t)=\exp -\frac{\left(I-x_{i+s, j+t}\right)}{2 \sigma^{2} R}
\end{array}
$$

\subsection{BDND Filter [14]}

If the pixel is considered as the impulsive noise it is cleared by using the improved BDND filter [14]. The Efficient BDND Filtering Algorithm is calculated as below:

$$
\begin{gathered}
F_{m n}=\text { medidan }\left\{B_{m-p, n-u} \mid(p, u) \varepsilon V^{\wedge} B_{m-p, n-u} \varepsilon Z_{c}\right. \\
\mathrm{V}=\left\{(\mathrm{p}, \mathrm{u}) \mid-\left(\mathrm{V}_{\mathrm{Y}}-1\right) / 2 \leq(p, u) \leq\left(V_{Y}-1\right) / 2\right\}
\end{gathered}
$$

If the pixel is considered as the Gaussian noise it is cleared by using the dynamic non local mean filter [11]. The dynamic non local mean filter is calculated as below

$$
\mathrm{U}\left(\mathrm{R}_{1} \mathrm{R}_{2}\right)=\left\{\begin{array}{cc}
\bar{G}\left(R_{1}, R_{2}\right)=S\left(R_{1}, R_{2}\right) R_{2} \\
\frac{0\left(R_{1}\right)}{0\left(R_{2}\right)} & K 1 \neq K 2 \text { and }\left|0\left(\mathrm{R}_{1}\right)-0\left(\mathrm{R}_{2}\right)\right| \\
\{1 & \text { otherwise }
\end{array}\right.
$$




\section{Experiments \& Results}

\subsection{Experimental Images:}

The performance of the algorithm is evaluated on several real images shown in Figure 1.1. These pictures are the most widely used standard test images used for image retargeting algorithms. These are till in the industry standard for tests. It is a good test images. These images are used for many image processing researches.

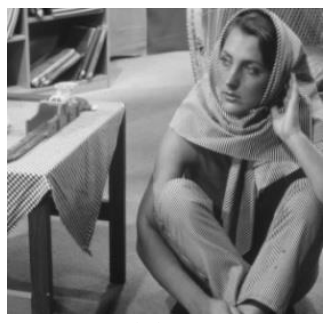

(a)

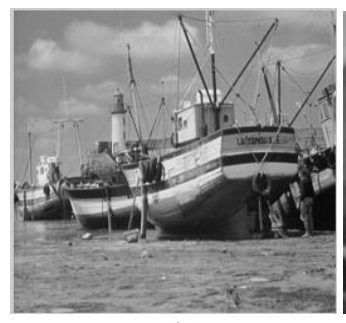

(b)

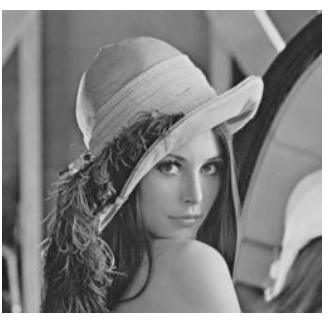

(c)

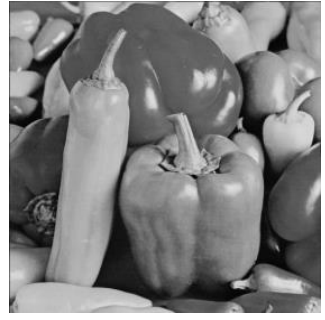

(d)

Figure 3.1.1. Experimental Images (a) Image of Barbra (b) Image of Sail Boat (c) Image of Lena (d) Image of Pepper

\subsection{Performance Metrics:}

To evaluate the performance of the denoising techniques several performance metrics are available. We use the Peak Signal-to-Noise Ratio, Mean Square Error and Structural Similarity Index Measure

1. Peak Signal-To-Noise-Ratio - Peak signal-to-noise ratio (PSNR) to evaluate the quality between the attacked image and the original image. The PSNR formula is defined as follows:

$$
\text { PSNR=10x } \log _{10} \frac{255 x 255}{\frac{1}{H x W} \sum_{x=0}^{H-1} \sum_{Y=0}^{W-1}[f(x, y)-g(x, y)]^{2}}
$$

where $\mathrm{H}$ and $\mathrm{W}$ are the height and width of the image, respectively; and $\mathrm{f}(\mathrm{x}, \mathrm{y})$ and $\mathrm{g}(\mathrm{x}, \mathrm{y})$ are the grey levels located at coordinate $(\mathrm{x}, \mathrm{y})$ of the original image and attacked image, respectively.

2. Mean Square Error:

The mean square error or MSE of an estimator is one of many ways to quantify the difference between an estimator and the true value of the quantity being estimated. As a loss function, MSE is called squared error loss.

$$
\mathrm{MSE}=\frac{\sum_{m=1}^{M} \sum_{n=1}^{N}\left[x(m, n)-x^{\prime}(m, n)\right]^{2}}{M * N}
$$

3. Structural similarity Index Measure

The Structural similarity Index Measure (SSIM) is a method for measuring the similarity between two images. The SSIM is given by,

$$
\operatorname{SSIM}(\mathrm{x}, \mathrm{y})=\frac{\left(2 \mu_{x} \mu_{y}+C_{1}\right)\left(2 \mu_{x y}+C_{2}\right)}{\left(\mu_{x}^{2}+\mu_{y}^{2}+C_{2}\right)\left(\sigma_{x}^{2}+\sigma_{y}^{2}+C_{2}\right)}
$$


4. Mean Absolute Error

The mean absolute error (MAE) is a quantity used to measure how difference between the original image and the noise free image. The mean absolute error is given by

$$
\text { MAE }=\frac{1}{n} \sum_{i=1}^{n}\left|f_{i}-y_{i}\right|
$$

5. Max Difference Value

The max difference value (MD) is a quantity used to measure how difference between the original image and the noise free image. The MD is given by

$$
\mathrm{MD}=\max \left(\left|f_{i}-y_{i}\right|\right)
$$

\subsubsection{Experimental Results}

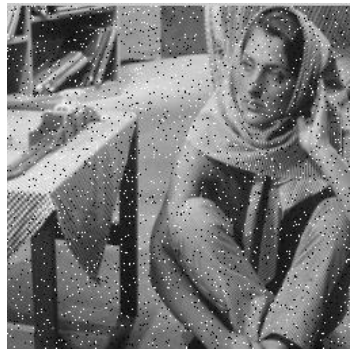

(a)

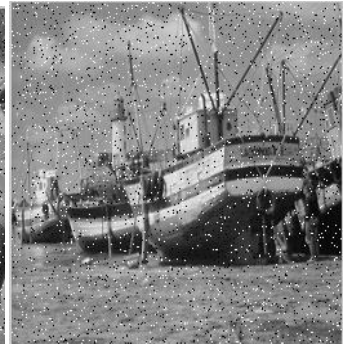

(b)

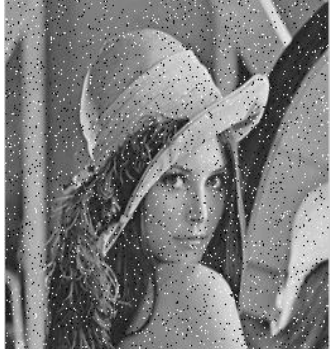

(c)

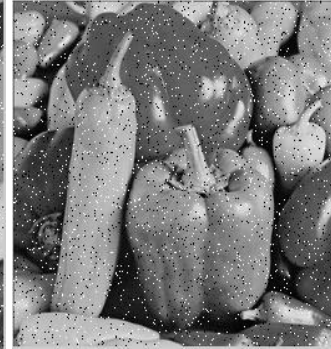

(d)

Figure 3.2.1.1. Impulse Noisy Images (a) Impulse Noisy Image of Barbara (b) Impulse Noisy Image of Sail Boat (c) Impulse Noisy Image of Lena (d) Impulse Noisy Image of Pepper

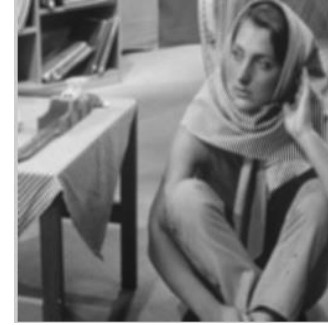

(a)

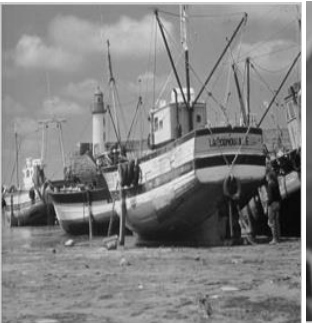

(b)

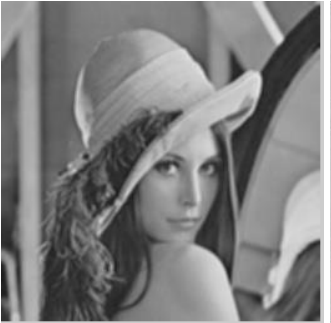

(c)

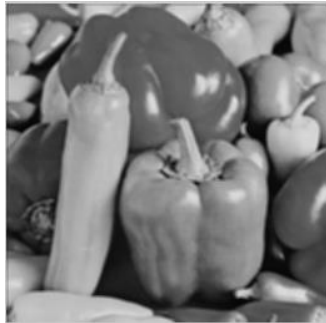

(d)

Figure 3.2.1.2. Noise Free Images using BDND Filter (a) Noise Free image of Barbara (b) Noise Free Image of Sail Boat (c) Noise Free image of Lena (d) Noise Free Image of Pepper

\subsection{Performance Analysis:}

The performance analysis of Impulse noise table has been evaluated, the Impulse noise ratio sigma $=5 \%$ to $50 \%$ iterations are evaluated for Barbara Image. Table 1 shows the performance of all techniques for Barbara Image.

Table 1. PERFORMANCE ANALYSIS OF Impulse Noise Table for Barbara Image

\begin{tabular}{|c|c|c|c|c|c|c|c|c|c|c|c|}
\hline Filte & Metric & \multicolumn{10}{|c|}{ Noise Ratio } \\
\cline { 3 - 11 } & $\mathrm{s}$ & $5 \%$ & $10 \%$ & $15 \%$ & $20 \%$ & $25 \%$ & $30 \%$ & $35 \%$ & $40 \%$ & $45 \%$ & $50 \%$ \\
\hline & & & & & & & & & & & 14.83 \\
& PSNR & 21.65 & 20.18 & 19.05 & 18.15 & 17.427 & 16.786 & 16.16 & 15.71 & 15.25 & 14.8319 \\
& & 32 & 22 & 92 & 42 & 6 & 8 & 18 & 16 & 08 & \\
\hline
\end{tabular}


International Journal of Signal Processing, Image Processing and Pattern Recognition Vol.7, No.5 (2014)

\begin{tabular}{|c|c|c|c|c|c|c|c|c|c|c|c|}
\hline \multirow[t]{4}{*}{$\mathrm{ABF}$} & MSE & $\begin{array}{c}28.61 \\
32 \\
\end{array}$ & $\begin{array}{c}29.97 \\
06 \\
\end{array}$ & $\begin{array}{c}30.41 \\
60 \\
\end{array}$ & $\begin{array}{c}31.53 \\
79 \\
\end{array}$ & $\begin{array}{c}34.289 \\
4 \\
\end{array}$ & $\begin{array}{c}36.915 \\
0 \\
\end{array}$ & $\begin{array}{c}39.66 \\
88 \\
\end{array}$ & $\begin{array}{c}41.77 \\
93 \\
\end{array}$ & $\begin{array}{c}44.05 \\
57 \\
\end{array}$ & 46.2323 \\
\hline & SSIM & $\begin{array}{c}0.606 \\
7 \\
\end{array}$ & $\begin{array}{c}0.606 \\
5 \\
\end{array}$ & $\begin{array}{c}0.605 \\
1 \\
\end{array}$ & $\begin{array}{c}0.604 \\
0\end{array}$ & 0.6029 & 0.6016 & $\begin{array}{c}0.600 \\
5\end{array}$ & $\begin{array}{c}0.599 \\
0\end{array}$ & $\begin{array}{c}0.597 \\
7 \\
\end{array}$ & 0.5392 \\
\hline & MAE & $\begin{array}{c}21.45 \\
25\end{array}$ & $\begin{array}{c}22.81 \\
62\end{array}$ & $\begin{array}{c}24.35 \\
86\end{array}$ & $\begin{array}{c}26.09 \\
88\end{array}$ & $\begin{array}{c}27.982 \\
3\end{array}$ & $\begin{array}{c}30.015 \\
7\end{array}$ & $\begin{array}{c}32.31 \\
20\end{array}$ & $\begin{array}{c}34.36 \\
31\end{array}$ & $\begin{array}{c}35.04 \\
74\end{array}$ & 36.5766 \\
\hline & MD & 235.1 & 235.3 & 235.8 & 236 & 239 & 240 & 243 & 243.7 & 244 & 244.2 \\
\hline \multirow{5}{*}{ FPGF } & $\begin{array}{l}\text { PSNR } \\
\end{array}$ & $\begin{array}{c}24.42 \\
84\end{array}$ & $\begin{array}{c}24.38 \\
76\end{array}$ & $\begin{array}{c}23.95 \\
2\end{array}$ & $\begin{array}{c}23.85 \\
77\end{array}$ & $\begin{array}{c}22.983 \\
0 \\
\end{array}$ & $\begin{array}{c}22.272 \\
2 \\
\end{array}$ & $\begin{array}{c}21.57 \\
43\end{array}$ & $\begin{array}{c}21.06 \\
65\end{array}$ & $\begin{array}{c}20.54 \\
12 \\
\end{array}$ & 20.0826 \\
\hline & MSE & $\begin{array}{c}26.18 \\
38 \\
\end{array}$ & $\begin{array}{c}27.73 \\
51 \\
\end{array}$ & $\begin{array}{c}28.63 \\
90 \\
\end{array}$ & $\begin{array}{c}29.08 \\
40\end{array}$ & $\begin{array}{c}32.126 \\
7\end{array}$ & $\begin{array}{c}34.908 \\
3 \\
\end{array}$ & $\begin{array}{c}37.82 \\
89 \\
\end{array}$ & $\begin{array}{c}40.13 \\
41 \\
\end{array}$ & $\begin{array}{c}42.60 \\
71\end{array}$ & 44.9168 \\
\hline & SSIM & $\begin{array}{c}0.719 \\
8 \\
\end{array}$ & $\begin{array}{c}0.719 \\
6 \\
\end{array}$ & $\begin{array}{c}0.719 \\
5\end{array}$ & $\begin{array}{c}0.709 \\
9 \\
\end{array}$ & 0.7071 & 0.6357 & $\begin{array}{c}0.621 \\
1\end{array}$ & $\begin{array}{c}0.609 \\
2\end{array}$ & $\begin{array}{c}0.608 \\
1\end{array}$ & 0.6020 \\
\hline & MAE & $\begin{array}{c}10.58 \\
84\end{array}$ & $\begin{array}{c}10.61 \\
89\end{array}$ & $\begin{array}{c}10.62 \\
43\end{array}$ & $\begin{array}{c}10.63 \\
99\end{array}$ & $\begin{array}{c}10.665 \\
0\end{array}$ & $\begin{array}{c}10.671 \\
8\end{array}$ & $\begin{array}{c}10.70 \\
32\end{array}$ & $\begin{array}{c}10.75 \\
71\end{array}$ & $\begin{array}{c}10.79 \\
62\end{array}$ & 10.8592 \\
\hline & MD & 230 & 230.3 & 230.8 & 231 & 233.2 & 234.1 & 235 & 235.8 & 236 & 239 \\
\hline \multirow{5}{*}{ SBF } & PSNR & $\begin{array}{c}22.41 \\
85 \\
\end{array}$ & $\begin{array}{c}21.46 \\
16 \\
\end{array}$ & $\begin{array}{c}21.42 \\
95 \\
\end{array}$ & $\begin{array}{c}20.62 \\
93 \\
\end{array}$ & $\begin{array}{c}20.006 \\
5 \\
\end{array}$ & $\begin{array}{c}19.428 \\
6 \\
\end{array}$ & $\begin{array}{c}18.92 \\
14 \\
\end{array}$ & $\begin{array}{c}15.42 \\
15 \\
\end{array}$ & $\begin{array}{c}15.01 \\
61 \\
\end{array}$ & 14.6487 \\
\hline & MSE & $\begin{array}{c}24.30 \\
04\end{array}$ & $\begin{array}{c}27.13 \\
07 \\
\end{array}$ & $\begin{array}{c}30.55 \\
38 \\
\end{array}$ & $\begin{array}{c}33.50 \\
25 \\
\end{array}$ & $\begin{array}{c}35.992 \\
8\end{array}$ & $\begin{array}{c}38.469 \\
0 \\
\end{array}$ & $\begin{array}{c}40.78 \\
20 \\
\end{array}$ & $\begin{array}{c}43.19 \\
83\end{array}$ & $\begin{array}{c}5.26 \\
20 \\
\end{array}$ & 47.2180 \\
\hline & SSIM & $\begin{array}{c}0.862 \\
1 \\
\end{array}$ & $\begin{array}{c}0.857 \\
2 \\
\end{array}$ & $\begin{array}{c}0.826 \\
0\end{array}$ & $\begin{array}{c}0.839 \\
4\end{array}$ & 0.8276 & 0.7978 & $\begin{array}{c}0.783 \\
1\end{array}$ & $\begin{array}{c}0.761 \\
8 \\
\end{array}$ & $\begin{array}{c}0.729 \\
7\end{array}$ & 0.6771 \\
\hline & MAE & $\begin{array}{c}12.83 \\
78\end{array}$ & $\begin{array}{c}12.87 \\
80\end{array}$ & $\begin{array}{c}12.94 \\
86\end{array}$ & $\begin{array}{c}12.10 \\
24\end{array}$ & $\begin{array}{c}12.107 \\
6\end{array}$ & $\begin{array}{c}12.117 \\
6\end{array}$ & $\begin{array}{c}12.13 \\
29\end{array}$ & $\begin{array}{c}12.20 \\
12\end{array}$ & $\begin{array}{c}12.48 \\
70\end{array}$ & 12.5103 \\
\hline & MD & 233.9 & 234.3 & 235.8 & $\begin{array}{c}235.2 \\
3\end{array}$ & 236.2 & 237.8 & 238 & 239 & 241 & 243 \\
\hline \multirow{5}{*}{$\begin{array}{l}\text { BDND } \\
\text { Filter }\end{array}$} & PSNR & $\begin{array}{c}32.32 \\
49 \\
\end{array}$ & $\begin{array}{c}32.15 \\
28 \\
\end{array}$ & $\begin{array}{c}31.00 \\
37 \\
\end{array}$ & $\begin{array}{c}30.19 \\
56 \\
\end{array}$ & $\begin{array}{c}30.432 \\
5 \\
\end{array}$ & $\begin{array}{c}29.267 \\
4 \\
\end{array}$ & $\begin{array}{c}29.89 \\
43 \\
\end{array}$ & $\begin{array}{c}28.18 \\
22 \\
\end{array}$ & $\begin{array}{c}27.73 \\
21 \\
\end{array}$ & 27.0340 \\
\hline & MSE & $\begin{array}{c}20.30 \\
24 \\
\end{array}$ & $\begin{array}{c}21.13 \\
12 \\
\end{array}$ & $\begin{array}{c}23.55 \\
31 \\
\end{array}$ & $\begin{array}{c}23.28 \\
25 \\
\end{array}$ & $\begin{array}{c}24.912 \\
8 \\
\end{array}$ & $\begin{array}{c}25.461 \\
6 \\
\end{array}$ & $\begin{array}{c}27.28 \\
20 \\
\end{array}$ & $\begin{array}{c}28.12 \\
09 \\
\end{array}$ & $\begin{array}{c}30.16 \\
21 \\
\end{array}$ & 32.1184 \\
\hline & SSIM & $\begin{array}{c}0.918 \\
9\end{array}$ & $\begin{array}{c}0.918 \\
7\end{array}$ & $\begin{array}{c}0.907 \\
1\end{array}$ & $\begin{array}{c}0.902 \\
7\end{array}$ & 0.9012 & 0.8990 & $\begin{array}{c}0.899 \\
3\end{array}$ & $\begin{array}{c}0.898 \\
8\end{array}$ & $\begin{array}{c}0.898 \\
4\end{array}$ & 0.8819 \\
\hline & MAE & $\begin{array}{c}8.237 \\
8\end{array}$ & $\begin{array}{c}8.378 \\
0\end{array}$ & $\begin{array}{c}8.448 \\
6\end{array}$ & $\begin{array}{c}8.528 \\
4\end{array}$ & 8.5576 & 8.6009 & $\begin{array}{c}8.611 \\
1\end{array}$ & $\begin{array}{c}8.712 \\
3\end{array}$ & $\begin{array}{c}8.890 \\
1\end{array}$ & 8.8988 \\
\hline & MD & 226 & 226.4 & 225 & 224 & 224.8 & 225.2 & 227.4 & 228.1 & 229.3 & 230 \\
\hline
\end{tabular}

The performance analysis of Impulse noise Table has been evaluated, the Impulse noise ratio sigma $=5 \%$ to $50 \%$ iterations are evaluated for Sail Boat Image. Table 2 shows performance of all techniques for Sail Boat Image.

Table 2. PERFORMANCE ANALYSIS OF Impulse Noise Table for Sail Boat Image

\begin{tabular}{|c|c|c|c|c|c|c|c|c|c|c|c|}
\hline \multirow{2}{*}{$\begin{array}{c}\text { Filter } \\
\mathrm{s}\end{array}$} & \multirow{2}{*}{$\begin{array}{l}\text { Metric } \\
\mathrm{s}\end{array}$} & \multicolumn{10}{|c|}{ Noise Ratio } \\
\hline & & $5 \%$ & $10 \%$ & $15 \%$ & $20 \%$ & $25 \%$ & $30 \%$ & $35 \%$ & $40 \%$ & $45 \%$ & $50 \%$ \\
\hline \multirow{5}{*}{$\mathrm{ABF}$} & PSNR & $\begin{array}{c}22.653 \\
1\end{array}$ & $\begin{array}{c}22.583 \\
2\end{array}$ & $\begin{array}{c}22.15 \\
92\end{array}$ & $\begin{array}{c}21.95 \\
41\end{array}$ & 21.7674 & 20.7828 & $\begin{array}{c}20.161 \\
8\end{array}$ & $\begin{array}{c}19.321 \\
0\end{array}$ & $\begin{array}{c}18.241 \\
0\end{array}$ & $\begin{array}{c}18.101 \\
8\end{array}$ \\
\hline & MSE & $\begin{array}{c}27.613 \\
2 \\
\end{array}$ & $\begin{array}{c}28.970 \\
6\end{array}$ & $\begin{array}{c}29.41 \\
60\end{array}$ & $\begin{array}{c}30.53 \\
99\end{array}$ & 31.2104 & 32.9150 & $\begin{array}{c}33.698 \\
8 \\
\end{array}$ & $\begin{array}{c}34.779 \\
3 \\
\end{array}$ & $\begin{array}{c}36.059 \\
8 \\
\end{array}$ & $\begin{array}{c}37.230 \\
1 \\
\end{array}$ \\
\hline & SSIM & 0.5067 & 0.5065 & $\begin{array}{c}0.505 \\
1\end{array}$ & $\begin{array}{c}0.504 \\
0\end{array}$ & 0.5029 & 0.5016 & 0.4915 & 0.4890 & 0.4877 & 0.4392 \\
\hline & MAE & $\begin{array}{c}23.452 \\
5\end{array}$ & $\begin{array}{c}23.816 \\
2\end{array}$ & $\begin{array}{c}23.35 \\
86\end{array}$ & $\begin{array}{c}23.09 \\
88\end{array}$ & 23.9823 & 26.0157 & $\begin{array}{c}27.312 \\
0\end{array}$ & $\begin{array}{c}28.363 \\
1\end{array}$ & $\begin{array}{c}29.047 \\
4\end{array}$ & $\begin{array}{c}31.576 \\
6\end{array}$ \\
\hline & MD & 238 & 239 & 239.8 & 239.9 & 240 & 240.6 & 243 & 243.7 & 244 & 244.2 \\
\hline \multirow{3}{*}{ FPGF } & PSNR & $\begin{array}{c}26.128 \\
4\end{array}$ & $\begin{array}{c}25.773 \\
2\end{array}$ & $\begin{array}{c}25.35 \\
21\end{array}$ & $\begin{array}{c}24.85 \\
00\end{array}$ & 24.1230 & 24.4561 & $\begin{array}{c}23.198 \\
7\end{array}$ & $\begin{array}{c}23.123 \\
5\end{array}$ & $\begin{array}{c}22.871 \\
2\end{array}$ & $\begin{array}{c}22.081 \\
2\end{array}$ \\
\hline & MSE & $\begin{array}{c}28.183 \\
8 \\
\end{array}$ & $\begin{array}{c}28.735 \\
1 \\
\end{array}$ & $\begin{array}{c}28.63 \\
90\end{array}$ & $\begin{array}{c}29.08 \\
40\end{array}$ & 29.1267 & 30.9083 & $\begin{array}{c}30.828 \\
9 \\
\end{array}$ & $\begin{array}{c}31.134 \\
1 \\
\end{array}$ & $\begin{array}{c}32.607 \\
1 \\
\end{array}$ & $\begin{array}{c}34.998 \\
8 \\
\end{array}$ \\
\hline & SSIM & 0.8901 & 0.8896 & 0.888 & 0.887 & 0.8861 & 0.8851 & 0.8751 & 0.8662 & 0.8081 & 0.6020 \\
\hline
\end{tabular}




\begin{tabular}{|c|c|c|c|c|c|c|c|c|c|c|c|}
\hline & & & & 5 & 9 & & & & & & \\
\hline & MAE & $\begin{array}{c}11.588 \\
4\end{array}$ & $\begin{array}{c}11.618 \\
9\end{array}$ & $\begin{array}{c}11.62 \\
43\end{array}$ & $\begin{array}{c}11.63 \\
99\end{array}$ & 11.6650 & 11.6718 & $\begin{array}{c}11.703 \\
2\end{array}$ & $\begin{array}{c}11.757 \\
1\end{array}$ & $\begin{array}{c}11.796 \\
2\end{array}$ & $\begin{array}{c}11.859 \\
2\end{array}$ \\
\hline & MD & 231 & 231.3 & 231.2 & 232 & 232.6 & 233.4 & 234 & 235.9 & 236.5 & 237 \\
\hline \multirow{5}{*}{ SBF } & PSNR & $\begin{array}{c}23.418 \\
5\end{array}$ & $\begin{array}{c}22.461 \\
2\end{array}$ & $\begin{array}{c}22.42 \\
91\end{array}$ & $\begin{array}{c}21.62 \\
32\end{array}$ & 21.0065 & 20.9286 & $\begin{array}{c}19.111 \\
4\end{array}$ & $\begin{array}{c}18.421 \\
1\end{array}$ & $\begin{array}{c}17.098 \\
7\end{array}$ & $\begin{array}{c}16.128 \\
7\end{array}$ \\
\hline & MSE & $\begin{array}{c}24.330 \\
4\end{array}$ & $\begin{array}{c}27.900 \\
7\end{array}$ & $\begin{array}{c}28.55 \\
38\end{array}$ & $\begin{array}{c}30.80 \\
25\end{array}$ & 32.1201 & 33.1210 & $\begin{array}{c}35.712 \\
0\end{array}$ & $\begin{array}{c}37.118 \\
2\end{array}$ & $\begin{array}{c}39.263 \\
0\end{array}$ & $\begin{array}{c}40.218 \\
0\end{array}$ \\
\hline & SSIM & 0.8910 & 0.8771 & $\begin{array}{c}0.866 \\
3\end{array}$ & $\begin{array}{c}0.859 \\
0\end{array}$ & 0.8475 & 0.8372 & 0.8231 & 0.7623 & 0.7298 & 0.6712 \\
\hline & MAE & $\begin{array}{c}12.837 \\
8\end{array}$ & $\begin{array}{c}12.878 \\
0\end{array}$ & $\begin{array}{c}12.94 \\
86\end{array}$ & $\begin{array}{c}12.10 \\
24\end{array}$ & 12.1076 & 12.1176 & $\begin{array}{c}12.132 \\
9\end{array}$ & $\begin{array}{c}12.201 \\
2\end{array}$ & $\begin{array}{c}12.487 \\
0\end{array}$ & $\begin{array}{c}12.510 \\
3\end{array}$ \\
\hline & MD & 230 & 231.4 & 232 & 233.1 & 234.8 & 235.2 & 237.4 & 238.1 & 239.3 & 240.9 \\
\hline \multirow{5}{*}{$\begin{array}{l}\text { BDND } \\
\text { Filter }\end{array}$} & PSNR & $\begin{array}{c}31.329 \\
9\end{array}$ & $\begin{array}{c}31.158 \\
7\end{array}$ & $\begin{array}{c}31.00 \\
37\end{array}$ & $\begin{array}{c}30.19 \\
56\end{array}$ & 29.9325 & 29.7074 & $\begin{array}{c}29.634 \\
3\end{array}$ & $\begin{array}{c}28.992 \\
2\end{array}$ & $\begin{array}{c}27.752 \\
1\end{array}$ & $\begin{array}{c}26.552 \\
3\end{array}$ \\
\hline & MSE & $\begin{array}{c}21.302 \\
4\end{array}$ & $\begin{array}{c}21.131 \\
2 \\
\end{array}$ & $\begin{array}{c}22.55 \\
31\end{array}$ & $\begin{array}{c}22.38 \\
25\end{array}$ & 23.9128 & 24.5016 & $\begin{array}{c}26.120 \\
9\end{array}$ & $\begin{array}{c}28.120 \\
9\end{array}$ & $\begin{array}{c}30.162 \\
3 \\
\end{array}$ & $\begin{array}{c}32.784 \\
3 \\
\end{array}$ \\
\hline & SSIM & 0.9189 & 0.9187 & $\begin{array}{c}0.907 \\
1\end{array}$ & $\begin{array}{c}0.902 \\
7\end{array}$ & 0.9012 & 0.8990 & 0.8993 & 0.8988 & 0.8984 & 0.8819 \\
\hline & MAE & 7.2378 & 7.3780 & $\begin{array}{c}7.448 \\
6\end{array}$ & $\begin{array}{c}7.524 \\
8\end{array}$ & 7.5567 & 7.6015 & 7.6101 & 7.8023 & 7.8918 & 7.8912 \\
\hline & MD & 225 & 225.4 & 226 & 226.3 & 226.8 & 227.2 & 227.4 & 227.9 & 228.3 & 229 \\
\hline
\end{tabular}

The performance analysis of Impulse noise Table has been evaluated, the Impulse noise ratio sigma $=5 \%$ to $50 \%$ iterations are evaluated for Lena Image. Table 3 shows performance of all techniques for Lena Image.

Table 3. PERFORMANCE ANALYSIS OF Impulse Noise Table for Lena Image

\begin{tabular}{|c|c|c|c|c|c|c|c|c|c|c|c|}
\hline \multirow{2}{*}{$\begin{array}{c}\text { Filter } \\
\mathrm{s}\end{array}$} & \multirow{2}{*}{$\begin{array}{l}\text { Metric } \\
\mathrm{s}\end{array}$} & \multicolumn{10}{|c|}{ Noise Ratio } \\
\hline & & $5 \%$ & $10 \%$ & $15 \%$ & $20 \%$ & $25 \%$ & $30 \%$ & $35 \%$ & $40 \%$ & $45 \%$ & $50 \%$ \\
\hline \multirow{5}{*}{$\mathrm{ABF}$} & PSNR & $\begin{array}{c}23.653 \\
1\end{array}$ & $\begin{array}{c}23.282 \\
2\end{array}$ & $\begin{array}{c}22.65 \\
93\end{array}$ & $\begin{array}{c}21.05 \\
49\end{array}$ & 21.3275 & 20.7112 & $\begin{array}{c}19.131 \\
8\end{array}$ & $\begin{array}{c}18.911 \\
1\end{array}$ & $\begin{array}{c}18.660 \\
8\end{array}$ & $\begin{array}{c}17.001 \\
9\end{array}$ \\
\hline & MSE & $\begin{array}{c}27.613 \\
9\end{array}$ & $\begin{array}{c}27.970 \\
8 \\
\end{array}$ & $\begin{array}{c}28.41 \\
67 \\
\end{array}$ & $\begin{array}{c}29.53 \\
76 \\
\end{array}$ & 30.2895 & 31.9154 & $\begin{array}{c}33.668 \\
3 \\
\end{array}$ & $\begin{array}{c}34.779 \\
2 \\
\end{array}$ & $\begin{array}{c}36.055 \\
0\end{array}$ & $\begin{array}{c}38.232 \\
1\end{array}$ \\
\hline & SSIM & 0.6967 & 0.8965 & $\begin{array}{c}0.675 \\
1\end{array}$ & $\begin{array}{c}0.684 \\
0\end{array}$ & 0.6429 & 0.6516 & 0.6905 & 0.5912 & 0.5910 & 0.5392 \\
\hline & MAE & $\begin{array}{c}22.435 \\
6\end{array}$ & $\begin{array}{c}23.098 \\
7\end{array}$ & $\begin{array}{c}23.23 \\
48\end{array}$ & $\begin{array}{c}23.98 \\
76\end{array}$ & 24.3315 & 25.1178 & $\begin{array}{c}26.660 \\
9\end{array}$ & $\begin{array}{c}27.112 \\
3\end{array}$ & $\begin{array}{c}28.678 \\
1\end{array}$ & $\begin{array}{c}29.091 \\
2\end{array}$ \\
\hline & MD & 235.1 & 235.3 & 235.8 & 236 & 239 & 240 & 243 & 243.7 & 244 & 244.2 \\
\hline \multirow{5}{*}{ FPGF } & PSNR & $\begin{array}{c}24.428 \\
4 \\
\end{array}$ & $\begin{array}{c}24.387 \\
6\end{array}$ & $\begin{array}{c}23.95 \\
2\end{array}$ & $\begin{array}{c}23.85 \\
77\end{array}$ & 22.9830 & 22.2722 & $\begin{array}{c}21.574 \\
3\end{array}$ & $\begin{array}{c}21.066 \\
5\end{array}$ & $\begin{array}{c}20.541 \\
2\end{array}$ & $\begin{array}{c}20.082 \\
6\end{array}$ \\
\hline & MSE & $\begin{array}{c}26.183 \\
8 \\
\end{array}$ & $\begin{array}{c}27.735 \\
1 \\
\end{array}$ & $\begin{array}{c}28.63 \\
90\end{array}$ & $\begin{array}{c}29.08 \\
40\end{array}$ & 32.1267 & 34.9083 & $\begin{array}{c}37.828 \\
9 \\
\end{array}$ & $\begin{array}{c}40.134 \\
1 \\
\end{array}$ & $\begin{array}{c}42.607 \\
1 \\
\end{array}$ & $\begin{array}{c}44.916 \\
8 \\
\end{array}$ \\
\hline & SSIM & 0.7198 & 0.7196 & $\begin{array}{c}0.719 \\
5\end{array}$ & $\begin{array}{c}0.709 \\
9\end{array}$ & 0.7071 & 0.6357 & 0.6211 & 0.6092 & 0.6081 & 0.6020 \\
\hline & MAE & $\begin{array}{c}10.588 \\
4\end{array}$ & $\begin{array}{c}10.618 \\
9\end{array}$ & $\begin{array}{c}10.62 \\
43\end{array}$ & $\begin{array}{c}10.63 \\
99\end{array}$ & 10.6650 & 10.6718 & $\begin{array}{c}10.703 \\
2\end{array}$ & $\begin{array}{c}10.757 \\
1\end{array}$ & $\begin{array}{c}10.796 \\
2\end{array}$ & $\begin{array}{c}10.859 \\
2\end{array}$ \\
\hline & MD & 230 & 230.3 & 230.8 & 231 & 233.2 & 234.1 & 235 & 235.8 & 236 & 239 \\
\hline \multirow{5}{*}{ SBF } & PSNR & $\begin{array}{c}25.918 \\
5 \\
\end{array}$ & $\begin{array}{c}25.861 \\
6\end{array}$ & $\begin{array}{c}25.62 \\
95\end{array}$ & $\begin{array}{c}25.32 \\
93\end{array}$ & 25.1065 & 24.8286 & $\begin{array}{c}23.621 \\
4\end{array}$ & $\begin{array}{c}22.921 \\
5 \\
\end{array}$ & $\begin{array}{c}21.316 \\
1\end{array}$ & $\begin{array}{c}20.658 \\
7 \\
\end{array}$ \\
\hline & MSE & $\begin{array}{c}21.921 \\
0 \\
\end{array}$ & $\begin{array}{c}21.671 \\
0 \\
\end{array}$ & $\begin{array}{c}21.55 \\
38 \\
\end{array}$ & $\begin{array}{c}22.90 \\
25 \\
\end{array}$ & 23.9921 & 24.4694 & $\begin{array}{c}25.780 \\
01 \\
\end{array}$ & $\begin{array}{c}26.118 \\
7 \\
\end{array}$ & $\begin{array}{c}27.138 \\
7 \\
\end{array}$ & $\begin{array}{c}29.006 \\
1 \\
\end{array}$ \\
\hline & SSIM & 0.8120 & 0.8373 & $\begin{array}{c}0.857 \\
1\end{array}$ & $\begin{array}{c}0.869 \\
7\end{array}$ & 0.8476 & 0.8078 & 0.7831 & 0.7618 & 0.7297 & 0.6771 \\
\hline & MAE & $\begin{array}{c}11.817 \\
8\end{array}$ & $\begin{array}{c}11.828 \\
0\end{array}$ & $\begin{array}{c}11.94 \\
36\end{array}$ & $\begin{array}{c}12.14 \\
24\end{array}$ & 12.1075 & 12.6176 & $\begin{array}{c}12.872 \\
9\end{array}$ & $\begin{array}{c}13.201 \\
2\end{array}$ & $\begin{array}{c}13.387 \\
1\end{array}$ & $\begin{array}{c}14.445 \\
6\end{array}$ \\
\hline & MD & 233.9 & 234.3 & 235.8 & $\begin{array}{c}235.2 \\
3\end{array}$ & 236.2 & 237.8 & 238 & 239 & 241 & 243 \\
\hline
\end{tabular}


International Journal of Signal Processing, Image Processing and Pattern Recognition Vol.7, No.5 (2014)

\begin{tabular}{|c|c|c|c|c|c|c|c|c|c|c|c|}
\hline \multirow{5}{*}{$\begin{array}{l}\text { BDND } \\
\text { Filter }\end{array}$} & PSNR & $\begin{array}{c}32.324 \\
9\end{array}$ & $\begin{array}{c}32.152 \\
8\end{array}$ & $\begin{array}{c}31.00 \\
37\end{array}$ & $\begin{array}{c}30.19 \\
56\end{array}$ & 30.4325 & 29.2674 & $\begin{array}{c}29.894 \\
3\end{array}$ & $\begin{array}{c}28.182 \\
2\end{array}$ & $\begin{array}{c}27.732 \\
1\end{array}$ & $\begin{array}{c}27.034 \\
0\end{array}$ \\
\hline & MSE & $\begin{array}{c}20.302 \\
4\end{array}$ & $\begin{array}{c}21.131 \\
2\end{array}$ & $\begin{array}{c}23.55 \\
31 \\
\end{array}$ & $\begin{array}{c}23.28 \\
25 \\
\end{array}$ & 24.9128 & 25.4616 & $\begin{array}{c}27.282 \\
0\end{array}$ & $\begin{array}{c}28.120 \\
9\end{array}$ & $\begin{array}{c}30.162 \\
1\end{array}$ & $\begin{array}{c}32.118 \\
4\end{array}$ \\
\hline & SSIM & 0.9189 & 0.9187 & $\begin{array}{c}0.907 \\
1\end{array}$ & $\begin{array}{c}0.902 \\
7\end{array}$ & 0.9012 & 0.8990 & 0.8993 & 0.8988 & 0.8984 & 0.8819 \\
\hline & MAE & 8.2378 & 8.3780 & $\begin{array}{c}8.448 \\
6\end{array}$ & $\begin{array}{c}8.528 \\
4\end{array}$ & 8.5576 & 8.6009 & 8.6111 & 8.7123 & 8.8901 & 8.8988 \\
\hline & MD & 226 & 226.4 & 225 & 224 & 224.8 & 225.2 & 227.4 & 228.1 & 229.3 & 230 \\
\hline
\end{tabular}

The performance analysis of Impulse noise table has been evaluated, the Impulse noise ratio sigma $=5 \%$ to $50 \%$ iterations are evaluated for Pepper Image. Table 4 shows performance of all techniques for Pepper Image.

Table 4. PERFORMANCE ANALYSIS OF Impulse Noise Table for Pepper Image

\begin{tabular}{|c|c|c|c|c|c|c|c|c|c|c|c|}
\hline \multirow{2}{*}{$\begin{array}{c}\text { Filter } \\
\mathrm{s}\end{array}$} & \multirow[t]{2}{*}{ Metrics } & \multicolumn{10}{|c|}{ Noise Ratio } \\
\hline & & $5 \%$ & $10 \%$ & $15 \%$ & $20 \%$ & $25 \%$ & $30 \%$ & $35 \%$ & $40 \%$ & $45 \%$ & $50 \%$ \\
\hline \multirow{5}{*}{$\mathrm{ABF}$} & PSNR & $\begin{array}{c}22.953 \\
9\end{array}$ & $\begin{array}{c}22.883 \\
1\end{array}$ & $\begin{array}{c}22.75 \\
98\end{array}$ & $\begin{array}{c}21.65 \\
42\end{array}$ & 21.5677 & 20.1823 & $\begin{array}{c}20.261 \\
7\end{array}$ & $\begin{array}{c}19.321 \\
4\end{array}$ & $\begin{array}{c}18.441 \\
6\end{array}$ & $\begin{array}{c}18.505 \\
8\end{array}$ \\
\hline & MSE & $\begin{array}{c}27.113 \\
2\end{array}$ & $\begin{array}{c}28.210 \\
6\end{array}$ & $\begin{array}{c}29.34 \\
60\end{array}$ & $\begin{array}{c}30.67 \\
99\end{array}$ & 31.1004 & 32.6750 & $\begin{array}{c}33.198 \\
8\end{array}$ & $\begin{array}{c}34.659 \\
3\end{array}$ & $\begin{array}{c}36.229 \\
8\end{array}$ & $\begin{array}{c}37.170 \\
1\end{array}$ \\
\hline & SSIM & 0.5167 & 0.5165 & $\begin{array}{c}0.515 \\
1\end{array}$ & $\begin{array}{c}0.514 \\
0\end{array}$ & 0.5129 & 0.5116 & 0.4215 & 0.4490 & 0.4677 & 0.4092 \\
\hline & MAE & $\begin{array}{c}23.123 \\
4\end{array}$ & $\begin{array}{c}23.091 \\
2\end{array}$ & $\begin{array}{c}23.56 \\
43\end{array}$ & $\begin{array}{c}23.87 \\
56\end{array}$ & 23.1235 & 26.6753 & $\begin{array}{c}27.128 \\
0\end{array}$ & $\begin{array}{c}28.345 \\
6\end{array}$ & $\begin{array}{c}29.213 \\
4\end{array}$ & $\begin{array}{c}31.987 \\
1\end{array}$ \\
\hline & MD & 238.5 & 239.2 & 239.8 & 239.9 & 240.3 & 240.6 & 241 & 242.7 & 243.2 & 244.1 \\
\hline \multirow{5}{*}{ FPGF } & PSNR & $\begin{array}{c}26.128 \\
4\end{array}$ & $\begin{array}{c}25.773 \\
2\end{array}$ & $\begin{array}{c}25.35 \\
21\end{array}$ & $\begin{array}{c}24.85 \\
00\end{array}$ & 24.1230 & 24.4561 & $\begin{array}{c}23.198 \\
7\end{array}$ & $\begin{array}{c}23.123 \\
5\end{array}$ & $\begin{array}{c}22.871 \\
2\end{array}$ & $\begin{array}{c}22.081 \\
2\end{array}$ \\
\hline & MSE & $\begin{array}{c}28.183 \\
8\end{array}$ & $\begin{array}{c}28.735 \\
1\end{array}$ & $\begin{array}{c}28.63 \\
90\end{array}$ & $\begin{array}{c}29.08 \\
40\end{array}$ & 29.1267 & 30.9083 & $\begin{array}{c}30.828 \\
9\end{array}$ & $\begin{array}{c}31.134 \\
1\end{array}$ & $\begin{array}{c}32.607 \\
1\end{array}$ & $\begin{array}{c}34.998 \\
8\end{array}$ \\
\hline & SSIM & 0.8901 & 0.8896 & $\begin{array}{c}0.888 \\
5\end{array}$ & $\begin{array}{c}0.887 \\
9\end{array}$ & $\begin{array}{l}0.8861 \\
\end{array}$ & 0.8851 & 0.8751 & 0.8662 & 0.8081 & 0.6020 \\
\hline & MAE & $\begin{array}{c}10.588 \\
4\end{array}$ & $\begin{array}{c}10.618 \\
9\end{array}$ & $\begin{array}{c}10.62 \\
43\end{array}$ & $\begin{array}{c}10.63 \\
99\end{array}$ & 10.6650 & 10.6718 & $\begin{array}{c}10.703 \\
2\end{array}$ & $\begin{array}{c}10.757 \\
1\end{array}$ & $\begin{array}{c}10.796 \\
2\end{array}$ & $\begin{array}{c}10.859 \\
2\end{array}$ \\
\hline & MD & 230 & 230.3 & 230.8 & 231 & 233.2 & 234.1 & 235 & 235.8 & 236 & 239 \\
\hline \multirow{5}{*}{ SBF } & PSNR & $\begin{array}{c}23.418 \\
5\end{array}$ & $\begin{array}{c}22.461 \\
2\end{array}$ & $\begin{array}{c}22.42 \\
91\end{array}$ & $\begin{array}{c}21.62 \\
32\end{array}$ & 21.0065 & 20.9286 & $\begin{array}{c}19.111 \\
4\end{array}$ & $\begin{array}{c}18.421 \\
1\end{array}$ & $\begin{array}{c}17.098 \\
7\end{array}$ & $\begin{array}{c}16.128 \\
7\end{array}$ \\
\hline & MSE & $\begin{array}{c}24.330 \\
4\end{array}$ & $\begin{array}{c}27.900 \\
7\end{array}$ & $\begin{array}{c}28.55 \\
38\end{array}$ & $\begin{array}{c}30.80 \\
25\end{array}$ & 32.1201 & 33.1210 & $\begin{array}{c}35.712 \\
0\end{array}$ & $\begin{array}{c}37.118 \\
2\end{array}$ & $\begin{array}{c}39.263 \\
0\end{array}$ & $\begin{array}{c}40.218 \\
0\end{array}$ \\
\hline & SSIM & 0.8910 & 0.8771 & $\begin{array}{c}0.866 \\
3\end{array}$ & $\begin{array}{c}0.859 \\
0\end{array}$ & 0.8475 & 0.8372 & 0.8231 & 0.7623 & 0.7298 & 0.6712 \\
\hline & MAE & $\begin{array}{c}12.837 \\
1\end{array}$ & $\begin{array}{c}12.878 \\
2\end{array}$ & $\begin{array}{c}12.94 \\
89\end{array}$ & $\begin{array}{c}12.10 \\
21\end{array}$ & 12.1036 & 12.1091 & $\begin{array}{c}12.133 \\
1\end{array}$ & $\begin{array}{c}12.200 \\
0\end{array}$ & $\begin{array}{c}12.421 \\
0\end{array}$ & $\begin{array}{c}12.521 \\
5\end{array}$ \\
\hline & MD & 231 & 232.4 & 232.8 & 233.5 & 234.1 & 234.7 & 235.4 & 236.1 & 237.3 & 239 \\
\hline \multirow{5}{*}{$\begin{array}{l}\text { BDND } \\
\text { Filter }\end{array}$} & PSNR & $\begin{array}{c}33.114 \\
9\end{array}$ & $\begin{array}{c}33.101 \\
2\end{array}$ & $\begin{array}{c}33.00 \\
31\end{array}$ & $\begin{array}{c}32.91 \\
56\end{array}$ & 32.1291 & 31.4614 & $\begin{array}{c}31.994 \\
6\end{array}$ & $\begin{array}{c}30.982 \\
2\end{array}$ & $\begin{array}{c}30.732 \\
1\end{array}$ & $\begin{array}{c}29.034 \\
0\end{array}$ \\
\hline & MSE & $\begin{array}{l}19.302 \\
4\end{array}$ & $\begin{array}{c}20.131 \\
2\end{array}$ & $\begin{array}{c}20.55 \\
31\end{array}$ & $\begin{array}{c}20.28 \\
25\end{array}$ & 20.9328 & 21.4646 & $\begin{array}{c}21.209 \\
1\end{array}$ & $\begin{array}{c}21.341 \\
0\end{array}$ & $\begin{array}{c}22.109 \\
8\end{array}$ & $\begin{array}{c}23.111 \\
0\end{array}$ \\
\hline & SSIM & 0.9181 & 0.9182 & $\begin{array}{c}0.907 \\
3\end{array}$ & $\begin{array}{c}0.902 \\
4\end{array}$ & 0.9015 & 0.8999 & 0.8998 & 0.8987 & 0.8986 & 0.8815 \\
\hline & MAE & 7.2318 & 7.3790 & $\begin{array}{c}7.428 \\
6\end{array}$ & $\begin{array}{c}7.528 \\
4\end{array}$ & 7.5536 & 7.6007 & 7.6141 & 7.7623 & 7.8501 & 7.8960 \\
\hline & MD & 225 & 224.4 & 224 & 223 & 223.8 & 224.2 & 224.4 & 225.1 & 226.3 & 229 \\
\hline
\end{tabular}


From the above Table 1-4 it is shown that the BDND filter method is considered as the best method for High Density Impulse Noise Removal Techniques for all standard Images. Because it has higher PSNR (see Table 1-4) and SSIM value with lower MSE.

\section{Conclusion}

In this paper, High Density Impulse noise detection and reduction techniques were implemented and the results were compared by using five performance parameters. They are Peak-Signal-to-Noise-Ratio, Mean Square Error and structural similarity index measure, Mean Absolute Error, and Maximum Difference. The experimental results show that the BDND denoising method performed well than the other methods.

\section{References}

[1] K. N. Plataniotis and A. N. Venetsanopoulos, "Color Image Processing and Applications," Berlin, Germany, Springer, (2000).

[2] C. Tomasi and R.Manduchi, "Bilateral filter for gray and color images," in Proc. IEEE Int. Conf. Computer Vision, (1998), pp. 839-846.

[3] M. Elad, "On the origin of bilateral filter and ways to improve it," IEEE Trans. Image Process., vol. 11, no. 10, (2002) October, pp. 1141-1151.

[4] P. Perona and J. Malik, "Scale-space and edge detection using anisotropic diffusion," IEEE Trans. Pattern Anal. Mach. Intell., vol. 12, no. 7, (1990) July, pp. 629-639.

[5] L. Lucchese and S. K. Mitra, "A new class of chromatic filters for color image processing", Theory and applications," IEEE Trans. Image Process., vol. 13, no. 4, (2004) April, pp. 534-548.

[6] H. L. Eng and K. K. Ma, "Noise adaptive soft-switching median filter," IEEE Trans. Image Process., vol. 10, no. 2, (2001) February, pp. 242-251.

[7] D. Van de Ville, M. Nachtegael, D. P. Van der Weken, W. I. Lemahieu and E. E. Kerre, "Noise reduction by fuzzy image filtering," IEEE Trans. Image Process., vol. 11, no. 4, (2001) April, pp. 429-436.

[8] S. Morillas, V. Gregori and A. Sapena, "Fuzzy bilateral filtering for color images," in Proc. Int. Conf. Image Analysis and Recognition, Lecture Notes in Computer Science, vol. 4141, (2006), pp. 138-145.

[9] S. Schulte, V. Witte, De and E. E. Kerre, "A fuzzy noise reduction method for colour images," IEEE Trans. Image Process., vol. 16, no. 5, (2007) May, pp. 1425-1436.

[10] S. Morillas, S. Schulte, T. Mélange, E. E. Kerre and V.Gregori, "A soft-switching approach to improve visual quality of colour image smoothing filters," in Proc. Advanced Concepts for Intelligent Vision Systems, (2007), vol. 4678.

[11] B. Zhang and J. P. Allebach, "Adaptive Bilateral Filter for Sharpness Enhancement and Noise Removal", IEEE Transaction on Image Processing, vol. 17, no. 5.

[12] S. Morillas, V. Gregori and A. Sapena, "Fuzzy peer Groups Reducing Mixed Gaussian-Impulse Noise From Color Images," IEEE Transaction on Image Processing, vol. 18, no. 7, (2009) July.

[13] C.-H. L. Jia-Shiuan, "Switching Bilateral Filter with a Texture/Noise Detector for Universal Noise Removal", IEEE Transaction on Image Processing, vol. 19, no. 9, (2010) September.

[14] F. Iyad, A. J. Rami, "Efficient Improvements on the BDND Filtering Algorithm for the Removal of HighDensity Impulse noise”, IEEE Transaction on Image Processing, vol. 22, no. 3, (2013) March.

\section{Authors}

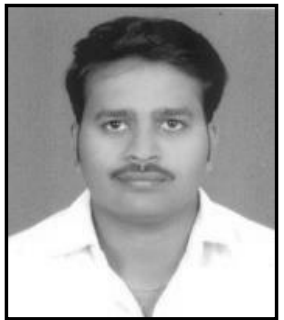

V. Murugan, he completed his M.C.A Degree from the Department of Computer Science and Engineering, Manonmaniam Sundaranar University, Tirunelveli in the year 2010.He has completed his M.E Degree from the Department of computer science and Engineering, Manonmaniam Sundaranar University, Tirunelveli in the year 2012. He is pursuing Research in the Department of Computer Science and Engineering, Manonmaniam Sundaranar University, Tirunelveli. His 
research interests include Image Enhancement and Parallel Image Processing.

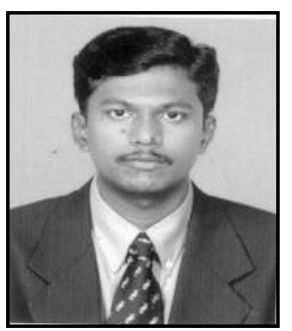

T. Avudaiappan, he completed his B.E Degree from the Department of Computer Science and Engineering, Jayaraj Annapackiam CSI college of Engineering from Anna University; Chennai in the year 2010.He has completed his M.E Degree from the Department of computer science and Engineering in Karpagam University, Coimbatore in the year 2012. He is a Research Scholar in the Department of Computer Science and Engineering, Manonmaniam Sundaranar University, Tirunelveli. His research interests include, Cloud Computing, Parallel Computing, Image Processing and Web Development.

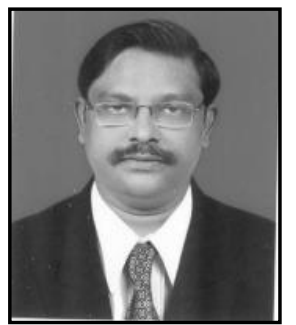

R. Balasubramanian, he received his B.E [Hons] degree in Computer Science and Engineering, from Bharathidhasan University in the year 1989. He completed his M.E degree in Computer Science and Engineering, from Regional Engineering College, Trichy/Bharathidhasan University in the year 1992. He is working as a Professor in the department of Computer Science and Engineering, Manonmaniam Sundaranar University,

Tirunelveli, he received his Doctorate in Computer Science and Engineering, Manonmaniam Sundaranar University, Tirunelveli in the field of Digital Image Processing, in the year 2011.He has published papers in many National and International Level Journals and Conferences. His research interests in the field of Digital Image Processing, Data mining, and Wireless Network \& Cloud Computing. 